\title{
Elevated expression of exosomal microRNA-21 as a potential biomarker for the early diagnosis of pancreatic cancer using a tethered cationic lipoplex nanoparticle biochip
}

\author{
XIAOFAN PU, GUOPING DING, MINGJIE WU, SENHAO ZHOU, SHENGNAN JIA and LIPING CAO \\ Department of General Surgery, Sir Run Run Shaw Hospital, School of Medicine, Zhejiang University, \\ Hangzhou, Zhejiang 310000, P.R. China
}

Received June 24, 2019; Accepted November 13, 2019

DOI: $10.3892 / \mathrm{ol} .2020 .11302$

\begin{abstract}
Pancreatic cancer (PC) has a poor prognosis due to the lack of effective molecular biomarkers for early diagnosis. Recent studies have investigated the use of exosomal microRNAs (exmiRs) as diagnostic biomarkers in cancer. The present study examined exmiR-21, exmiR-10b and exmiR-212-3p expression in patients with PC and healthy individuals. The expression levels of exmiR-21, exmiR-10b and exmiR-212-3p were examined in the peripheral blood plasma of 36 patients with PC and 65 healthy controls, using tethered cationic lipoplex nanoparticle biochip. The levels of exmiR-21 in the plasma of 34 mice were also evaluated. The expression levels of exmiR-21 and exmiR-10b were significantly greater in patients with PC compared with the control group. The receiver operating characteristic (ROC) analysis indicated that exmiR-21 had better diagnostic performance $(\mathrm{P}=0.0003$; $\mathrm{AUC}$, 0.7171) compared with the other two exmiRs. The diagnostic value of exmiR-21 improved when combined with exmiR-10b $(\mathrm{P}<0.0001$; AUC, 0.791). Furthermore, exmiR-21 was capable of distinguishing patients with early-stage $\mathrm{PC}$ from controls and advanced-stage $\mathrm{PC}(\mathrm{P}<0.05$, early stage vs. healthy; $\mathrm{P}<0.001$, early stage vs. advanced stage). The results of the present study revealed that the plasma levels of exmiR-21 and exmiR-10b were upregulated in patients with PC. The ROC analyses indicated that exmiR-21 had the best diagnostic performance among the three exmiRs. Furthermore, exmiR-21 was capable of discriminating patients with early-stage PC from healthy controls. These findings indicate the potential of determining the expression of exmiR-21 from serum using a tethered
\end{abstract}

Correspondence to: Professor Liping Cao or Dr Shengnan Jia, Department of General Surgery, Sir Run Run Shaw Hospital, School of Medicine, Zhejiang University, 3 East Qingchun Road, Hangzhou, Zhejiang 310000, P.R. China

E-mail: caolipingzju@zju.edu.cn

E-mail: jiashengnan8180@zju.edu.cn

Key words: exosomal microRNA-21, pancreatic cancer, early diagnosis, tethered cationic lipoplex nanoparticle biochip, biomarker cationic lipoplex nanoparticle biochip as a novel non-invasive strategy for the early diagnosis of PC.

\section{Introduction}

Pancreatic cancer (PC) is one of the most devastating types of cancer worldwide. As the third leading cause of cancer-associated mortality in the USA, PC was estimated to cause 44,330 deaths in this country in 2018 (1). The number of PC cases is projected to increase, and to become the second leading cause of cancer-associated mortality before 2030 (2).

Due to the anatomical location of PC, it is usually asymptomatic in the early stages of disease. However, without the use of invasive procedures, current screening methods are unable to detect early-stage PC (3), and these procedures are difficult to perform at the early stage of disease. Thus, the development of non-invasive detection methods, such as liquid biopsy, has been increasingly emphasized in recent years, and the identification of new biomarkers that can distinguish early stage PC is urgently needed (4).

MicroRNAs (miRs) play critical roles in a number of biological processes, such as cell proliferation, cell differentiation and apoptosis, through their regulation of gene expression by translational repression or mRNA degradation, resulting in gene silencing. Recent studies have indicated that miRs may also function as potential biomarkers for cancer detection (5-8). Exosomes are a type of extracellular vesicle, which are enclosed by a lipid bilayer that can contain various components, including proteins, lipids, DNA, mRNAs, miRs and non-coding RNAs (9). A number of studies have demonstrated that miRs contained within exosomes (exmiRs) play an important role in the development, metastasis and drug resistance of tumors (10-12). The lipid bilayer structure of exosomes prevents the enclosed miRs from degradation, allowing the detection of accurate numbers of stable exmiRs. Thus, isolating exosomes and quantifying the encapsulated exmiRs has become a promising approach for the noninvasive detection of cancer biomarkers (13).

miRs can be quantitatively measured in serum by reverse transcription-quantitative (RT-q)PCR. However, this approach is expensive and time-consuming (14). The tethered cationic lipoplex nanoparticle (TCLN) biochip is a novel technology 
for the direct analysis of exmiRs within a single exosome. Molecular beacons in liposome nanoparticles serve as molecular probes for target miRs. When serum is applied to the biochip, the molecular beacons recognize target miRs in exosomes, and the interaction is read by a total internal reflection fluorescence microscope (15). The copy numbers of the exmiRs can then be obtained by the fluorescence signals of the molecular beacons (MBs) (15). Wu et al (13) demonstrated that the levels of exmiRs detected by the TCLN biochip were consistent with the widely used reverse transcription-quantitative PCR (RT-qPCR) method. In addition, the TCLN biochip indicated a higher sensitivity compared with RT-qPCR (13).

The aim of the present study was to evaluate the expression levels of circulating exmiR-21, exmiR-10b and exmiR-212-3p between patients with PC and healthy individuals using a TCLN biochip and to determine whether these exmiRs can distinguish early-stage PC.

\section{Materials and methods}

Patients and clinical samples. A total of 36 patients with $\mathrm{PC}$ and 65 healthy individuals were enrolled for the present study between November 2017 and December 2018. The 36 patients with PC were divided into two groups, early- and advanced-stage; patients with stages I and II disease were categorized as the early stage group $(n=19)$, whereas patients with stages III and IV disease were categorized in the advanced-stage group $(n=17)$. All participants were from the Sir Run Run Shaw hospital of Zhejiang University (Zhejiang, China). The detailed characteristics of the patients are provided in Table I. All patients were newly diagnosed with PC, which was confirmed by two blinded pathologists from the Sir Run Run Shaw hospital, following surgery or ultrasound endoscopic guided fine needle aspiration biopsy. The blood samples of patients treated with chemotherapy, radiotherapy or immunotherapy were excluded. Tumors were staged according to the $8^{\text {th }}$ American Joint Committee on Cancer (AJCC) TNM Staging of Pancreatic Cancer (16).

The blood samples included in the present study were collected in vacuum blood tubes with EDTA anticoagulant before surgery and pharmacotherapy, and handled within $1 \mathrm{~h}$ following collection. The blood samples were subjected to centrifugation at $2,795 \times \mathrm{g}$ for $10 \mathrm{~min}$ at $4^{\circ} \mathrm{C}$. The plasma was then stored at $-80^{\circ} \mathrm{C}$. Written informed consent was obtained from the participants before sampling. The studies were conducted in accordance with the International Ethical Guidelines for Biomedical Research Involving Human Subjects (CIOMS), and the research protocols were approved by the Clinical Research Ethics Committee of Sir Run Run Shaw Hospital of Zhejiang University (Zhejiang, China)

Animal experiments. A total of 34 mice were obtained from the animal unit of Zhejiang University (Zhejiang, China). BALB/C nude mice (4-6 weeks old) were used in all experiments. All mice were female, and their weight ranged from $20-26 \mathrm{~g}$. The housing temperature was from $22-24^{\circ} \mathrm{C}$, and mice were given access to food and water. The air remained clean by using ventilation devices for $\sim 8-15$ times per hour, and the indoor lighting was controlled for $12 \mathrm{~h}$ of light and dark. All animal experiments were performed in the animal unit of
Zhejiang University according to procedures authorized and specifically approved by the institutional Ethical Committee. Subcutaneous xenograft mouse model were established in 12 mice by injecting $1 \times 10^{6}$ PANC-1-Luciferase cells (Stem Cell Bank, Chinese Academy of Science) in the shoulders, via a left subcostal incision, made in mice that were anesthetized with $3 \%$ isoflurane. The other 22 normal mice were used as the normal control and were injected with the same volume of normal saline. Subsequently, the blood of the 34 mice was collected, when the subcutaneous tumors in the 12 xenograft mice grew to a longitudinal diameter of $1 \mathrm{~cm}$. Blood was collected from the venous plexus of the eye socket, in mice anesthetized with $3 \%$ isoflurane, and the plasma was then separated by centrifugation at $2,795 \mathrm{x}$ g for $10 \mathrm{~min}$ at $4^{\circ} \mathrm{C}$. The plasma was then stored at $-80^{\circ} \mathrm{C}$. All mice were sacrificed by cervical dislocation under $3 \%$ isoflurane anesthesia, following the collection of blood. The plasma samples of these mice were used for further analysis.

Rationale for selecting exmiRs. As an oncomiR, a number of studies have demonstrated that miR-21 plays an important role in cell proliferation, migration, invasion and survival by regulating the cell cycle, apoptosis and invasion-related genes (17-21). Previous studies have proven that exosomal miR-21 is upregualted at the early stage of lung cancer, breast cancer, liver cancer and other malignant tumors (22-24).

Thus, exmiR-21 was considered as a potential biomarker for the diagnosis of cancer in the present study. However, to the best of our knowledge, few studies have focused on the assocaition between exmiR-21 and PC, particularly at the early stage of PC. miR-10b was associated with the invasive and metastatic properties of various human cancer types. Kim et al (25) identified that miR-10b promotes breast cancer cell proliferation, migration and invasion via the inhibition of transcription factor TBX5 expression, leading to the repression of the tumor suppressor genes (dual specificity tyrosine phosphorylation regulated kinase $1 \mathrm{~A}$ and PTEN). Previous studies regarded miR-10b as a potential diagnostic biomarker of breast cancer, bladder cancer and lung cancer (26). Ayaz et al (27) reported that miR-212-3p was specifically expressed in laryngeal squamous cell carcinoma. Furthermore, a study revealed that miR-212-3p was upregulated in PC tissue. However, to the best of our knowledge, few studies have focused on exmiRs in the aforementioned cancer types. Given that, exmiRs (particularly tumor-derived exmiRs) play an important role in the early diagnosis of several types of cancer in humans.

Preparing cationic lipoplex nanoparticles containing MBs. Firstly, miR-21-, miR-10b- and miR-212-3p-specific probes with fluorescent signal markers were designed. BHQ1 and 6-carboxy-fluorescein were assembled at the 3 ' and $5^{\prime}$ ends of the probe, respectively. The center of the probe is a specially designed single-stranded base sequence for identifying the target RNA. The specific molecular probe is coated in the cationic liposome nanoparticles and linked to the glass substrate to construct a PC-associated miRNA liposome nanoparticle chip. The probe sequences were as follows: MiR-21-MB, 5'-6FAM-TCAACATCAGTCTGATAAGCT ATTATCAGACTGA-BHQ1-3'; miR-10b-MB, 5'-6FAM-A TACCACACAAATTCGGTTCTACAACCGAATTTG 
Table I. Characteristics of patients with pancreatic cancer.

\begin{tabular}{|c|c|}
\hline Characteristics & Cases, n $(\%)$ \\
\hline \multicolumn{2}{|l|}{ Sex } \\
\hline Female & $15(41.7)$ \\
\hline Male & $21(58.3)$ \\
\hline \multicolumn{2}{|l|}{ Age (years) } \\
\hline$<60$ & $8(22.2)$ \\
\hline $60-69$ & $19(52.8)$ \\
\hline$\geq 70$ & $9(25.0)$ \\
\hline \multicolumn{2}{|c|}{ Cigarette smoking } \\
\hline No & $28(77.8)$ \\
\hline Yes & $8(22.2)$ \\
\hline \multicolumn{2}{|l|}{ Alcohol drinking } \\
\hline No & $7(19.4)$ \\
\hline Yes & $29(80.6)$ \\
\hline \multicolumn{2}{|c|}{ Diabetes mellitus } \\
\hline No & $33(91.7)$ \\
\hline Yes & $3(8.3)$ \\
\hline \multicolumn{2}{|l|}{ ABO blood type } \\
\hline A & $13(36.1)$ \\
\hline B & $8(22.2)$ \\
\hline $\mathrm{O}$ & $12(33.3)$ \\
\hline $\mathrm{AB}$ & $3(8.3)$ \\
\hline \multicolumn{2}{|l|}{ Stage } \\
\hline $\mathrm{I}$ & $6(16.7)$ \\
\hline II & $13(36.1)$ \\
\hline III & $5(13.9)$ \\
\hline IV & $12(33.3)$ \\
\hline \multicolumn{2}{|l|}{ BMI } \\
\hline$<19.57$ & $8(22.2)$ \\
\hline $19.57-23.71$ & $19(52.8)$ \\
\hline$>23.71$ & $9(25.0)$ \\
\hline
\end{tabular}

Continuous variables are converted into categorical variables. All variables are displayed as numbers and percentages. BMI, body mass index.

TG-BHQ1-3'; miR-212-3p-MB, 5'-6FAM-CGGCCGTGAC TGGAGACTGTTAAGTCTCCAGTCA-BHQ1-3'.

Exosome characterization and quantification. A commercial exosome extraction kit (Total Exosome Isolation kit; cat. no. 4484450; Invitrogen; Thermo Fisher Scientific, Inc.) was used to extract mouse serum and human plasma exosomes to detect their particle size and potential. The dynamic light scattering (DLS) technology was used for detection, with the use of a $640 \mathrm{~nm}$ laser and 30 frames per sec, which is also known as Photon Correlation Spectroscopy (PCS) or quasi-elastic scattering, is a method for measuring the variation of light intensity fluctuations over time (28). The Zetasizer Nano ZS90 instrument (Malvern Instruments, Inc.) was used for particle size analysis. The DLS technology has the advantages of accuracy, speediness and good repeatability, and has become a more conventional characterization method in nanotechnology (29). DLS is widely used, including for particle science testing in the life science field; it is mature, stable and credible. As a gold standard, it is recognized by the US Bureau of Standards and incorporated into the US measurement standard system. The calculation method of DLS should be able to reflect the particle size distribution within the system better.

Western blotting. Cells or exosomes were lysed with RIPA buffer (Beyotime Institute of Biotechnology), supplemented with protease and phosphatase inhibitor cocktail (Beyotime Institute of Biotechnology) on ice for $15 \mathrm{~min}$. The lysates were centrifuged at $12,000 \times \mathrm{g}$ for $5 \mathrm{~min}$ to pellet cell debris at $4^{\circ} \mathrm{C}$. The protein concentrations were measured by the BCA protein assay kit (Pierce; Thermo Fisher Scientific, Inc.), according to the manufacturer's instructions. Subsequently, the supernatant was added to SDS/PAGE sample loading buffer (Beyotime Institute of Biotechnology) and boiled for $10 \mathrm{~min}$ at $95^{\circ} \mathrm{C}$. A total mass of $20 \mu \mathrm{g}$ protein was run on SDS-PAGE (10\%) and transferred to polyvinylidene fluoride membrane (Bio-Rad Laboratories, Inc.). The membranes were blocked for $60 \mathrm{~min}$ in 5\% (w/v) non-fat dry milk or bovine serum albumin for $1 \mathrm{~h}$ at room temperature and incubated with primary antibodies overnight at $4^{\circ} \mathrm{C}$. The next day, the membranes were washed thrice in tris-buffered saline containing $0.1 \%$ (v/v) Tween-20 (TBST), incubated with horseradish-peroxidase conjugated secondary antibodies (Thermo Fisher Scientific, Inc.) for $1 \mathrm{~h}$ at room temperature, and then washed thrice in TBST. Bands were visualized using ChemiDoc Touch Imaging System (Bio-Rad Laboratories, Inc.) and the band intensities were quantified using the Image Lab 5.2.1 software (Bio-Rad Laboratories, Inc.). The primary antibodies were: Anti-CD9 (1:2,000; cat. no. ab92726; Abcam), anti-CD63 (1:1,000; cat. no. ab134045; Abcam) and anti-heat shock protein 70 (HSP70; 1:1,000; cat. no. ab181606; Abcam). The secondary antibody was: Goat anti-rabbit $\operatorname{IgG}(\mathrm{H}+\mathrm{L})$ secondary antibody, HRP (1:10,000; cat. no. 31460; Invitrogen; Thermo Fisher Scientific, Inc.)

Transmission electron microscopy (TEM). To evaluate the morphology of isolated exosomes, the exosomes were observed under a transmission electron microscope. Firstly, carbon-coated 400-mesh copper grids (Xinxing Bairui Co. Ltd.) were absorbed in a 20- $\mu$ l aliquot of exosomes $(\sim 2 \mu \mathrm{g})$ for $2 \mathrm{~min}$ and allowed to dry at room temperature. Subsequently, the exosomes were fixed in $1 \%(\mathrm{v} / \mathrm{v})$ glutaraldehyde (EM-grade) for $5 \mathrm{~min}$ at room temperature by placing drops carefully on the dried preparation. Next, the grids were washed twice with water for $5 \mathrm{~min}$ and then contrast-stained with $2 \%$ uranyl acetate for $10 \mathrm{~min}$ at room temperature. Images were obtained by transmission electron microscopy (Tecnai T10; FEI).

Statistical analysis. Statistical analyses were performed and graphs were constructed using GraphPad Prism (version 7.0; GraphPad Software, Inc.) and SPSS (version 24.0; IBM Corp.) statistical software. The data are presented as the mean \pm standard deviation. Significant differences between the mean values of two groups were determined using the Student's t-test. Significant differences between the mean values of three or four groups were determined using the one-way 
ANOVA, followed by Student-Newman-Keuls for further multiple comparisons. Clinicopathological diagnoses were used as the gold standard to assess diagnostic accuracy, using the receiver operating characteristic (ROC) curves generated with the GraphPad Prism 7.0 software. All diagnostic metrics (sensitivity and specificity) were calculated using the standard formulae (30). $\mathrm{P}<0.05$ was considered to indicate a statistically significant difference.

\section{Results}

Exosome characterization. DLS was used to detect the size of particles. Based on the DLS analysis, purified particles had a size of $53.5 \mathrm{~nm}$ in human plasma samples and $139.9 \mathrm{~nm}$ in mouse plasma samples, which was in accordance with the described view that exosomes are spherical particles with sizes ranging between 30 and $150 \mathrm{~nm}$ (31). The $\zeta$ potential values of plasma exosomes from both humans and mice were negative, -5.80 and $-1.49 \mathrm{mV}$ (Fig. 1A and D), respectively. CD9, CD63 and HSP70 are considered as exosomal-specific markers; the presence of these markers in the purified particles was assessed to characterize the purified particles as exosomes. Western blot analysis demonstrated that the purified particles expressed these markers, and thus could be considered as exosomes (Fig. 1B and E). Additionally, electron micrographs showed that the collected products had a distinctive cup shape (Fig. 1C and F). Overall, DLS, western blot analyses and electron micrographs confirmed that the particles tested were exosomes. Furthermore, as shown in Fig. 1G, the fluorescence intensity distributions indicated that more exosomes with higher exmiR-21, exmiR-10b and exmiR-212-3p expression were present in the samples of patients compared with those from healthy controls. Furthermore, murine PC exosomes revealed much higher fluorescence signals compared with plasma exosomes from healthy mouse (Fig. 1H).

Differential exmiR expression in patients with $P C$ and in healthy controls. The expression levels of exmiR-21, exmiR-10b and exmiR-212-3p were examined in the plasma of 36 patients with PC and 65 healthy individuals. The results showed that the expression levels of exmiR-21 and exmiR-10b were significantly increased in the plasma of patients with PC compared with the healthy control group (Fig. 2A and B) However, no significant difference $(\mathrm{P}=0.0698)$ was detected in the expression of exmiR-212-3p between the two groups (Fig. 2C).

ROC curve analysis. In order to evaluate the diagnostic performance of the three exmiRs, the ROC curve analysis was performed. The results revealed that exmiR-21 and exmiR-10b, but not exmiR-212-3p, could differentiate patients with PC from healthy individuals (Fig. 2D and E). The exmiR with the greatest area under the curve (AUC) was exmiR-21 ( $\mathrm{P}=0.0003$; AUC, 0.7171); the AUC of exmiR-10b was $0.6543(\mathrm{P}=0.0105)$. The ROC analysis indicated that the diagnostic accuracy of exmiR-21 was superior to the other miRs. ExmiR-21 and exmiR-10b were also combined in further ROC analysis, which indicated that exmiR-10b could improve the diagnostic performance of exmiR-21 $(\mathrm{P}<0.0001$; AUC, 0.791; Fig. 2).
ExmiR-21 was significantly upregulated in mice with PC and is a sensitive diagnostic marker. The expression of exmiR-21 was also examined using the TCLN biochip in the plasma of 12 mice with PC and 22 healthy mice. The level of exmiR-21 in the plasma of mice with PC was significantly higher $(\mathrm{P}=0.0001)$ compared with the healthy controls (Fig. 3A). The AUC of exmiR-21 in mice was 0.8636 ( $\mathrm{P}=0.0005$; Fig. 3B).

ExmiR-21 was significantly higher in patients with early-stage PC compared with healthy controls. The 36 patients with PC were stratified to examine the associations between the plasma levels of the three exmiRs and the PC stage, based on TNM staging. Detecting PC at an early stage (stages I or II) (32) may provide a window of opportunity when the disease can be adequately treated and stopped (33). The levels of exmiR-21 were significantly different in the early-stage group compared with the healthy control and patients with advanced-stage PC (Fig. 2F). In contrast, the expression levels of exmiR-10b and exmiR-212-3p showed no significant differences between the early-stage and the control groups (Fig. 2G and H).

Evaluating levels of the selected exmiRs with clinical parameters of patients with PC. Additionally, various clinical characteristics of the 36 patients with $\mathrm{PC}$, such as age, sex, history of smoking or drinking, diabetes mellitus, blood type, cancer stage and body mass index were analyzed. However, the levels of the three exmiRs showed no significant differences according to these characteristics (Fig. 4).

\section{Discussion}

One of the most important causes of the high mortality of patients with PC is the lack of robust and sensitive biomarkers for early diagnosis. The majority of patients with PC are diagnosed at a stage too late for surgical treatment (32). Thus, the development of new diagnostic methods that can detect early-stage PC is necessary to improve the survival of patients with PC.

The present study demonstrated that exmiR-21 and exmiR-10b were expressed at significantly higher levels in patients with PC compared with the healthy controls. Among the three exmiRs, exmiR-21 demonstrated the optimal diagnostic performance and, when combined with exmiR-10b, the diagnostic performance of exmiR-21 increased. The plasma of 34 mice with PC were also examined, which demonstrated elevated levels of exmiR-21 expression compared with controls. Furthermore, the expression of the three exmiRs in patients with early- or late-stage PC was also investigated in the present study. The results indicated that exmiR-21 level may predict the stage of PC and suggested that elevated exmiR-21 expression may serve as a potential biomarker for the early diagnosis of PC.

Numerous studies have reported an association between the overexpression of miR-21 and miR-10b in the plasma of patients with PC $(34,35)$. miR-21 and miR-10b have been associated with multiple cancer types, such as gastric and esophageal cancer (36), lung cancer (37) and breast cancer (38). However, few studies have examined exmiRs in plasma samples of patients with PC. Several studies have reported that exmiR-21 $(4,39)$ and exmiR-10b $(39,40)$ were significantly 
A

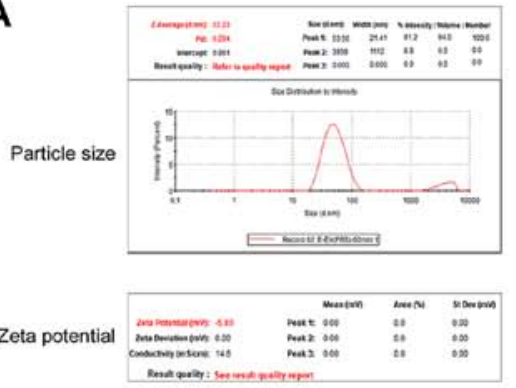

D

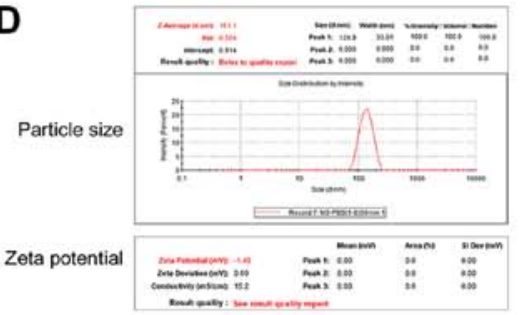

G

PC patient serum exosome
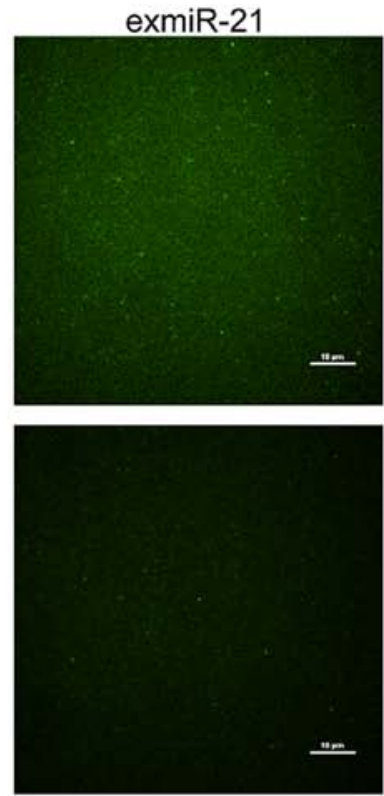

B

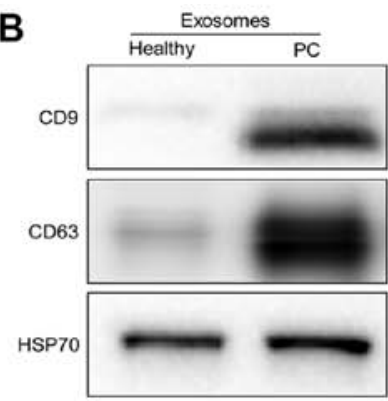

E

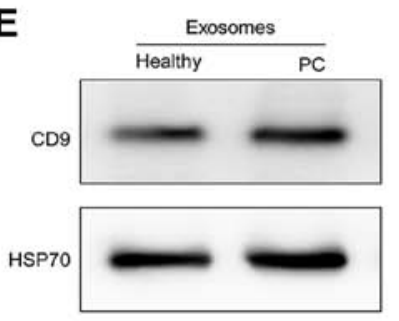

exmiR-10b
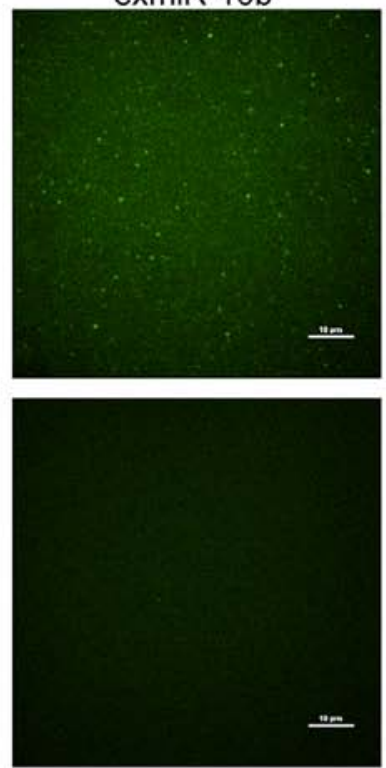

C

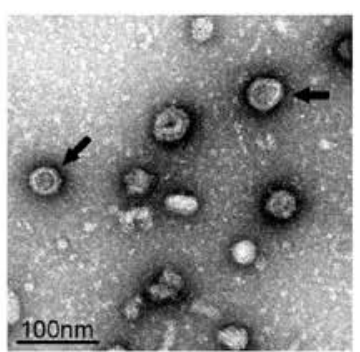

$\mathbf{F}$

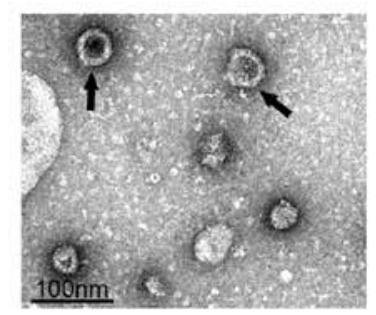

exmiR-212-3p
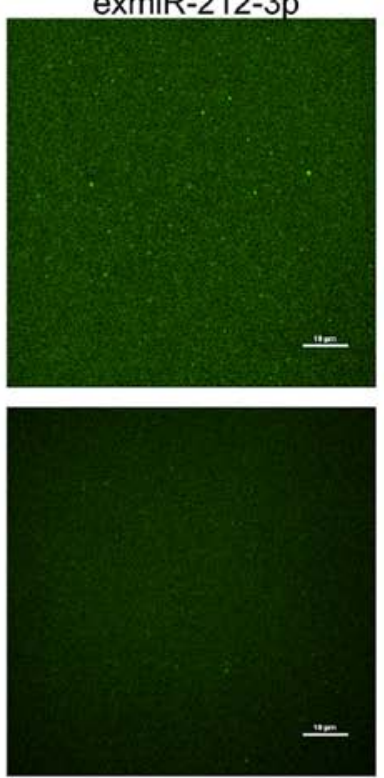

H

exmiR-21

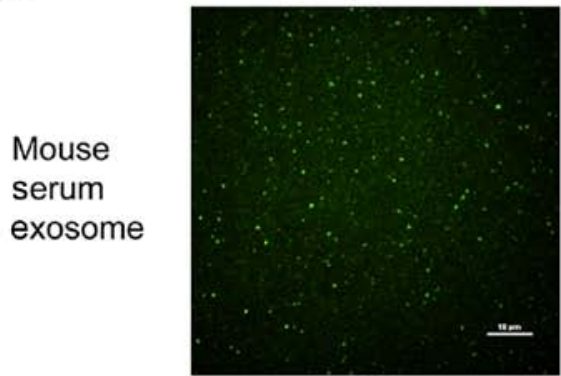

PC

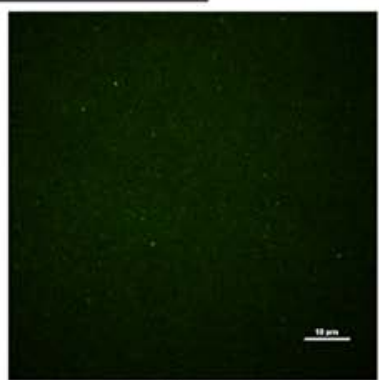

Normal

Figure 1. Characterization and quantification of exosome levels. (A) The particle size of exosomes in human plasma samples was $53.5 \mathrm{~nm}$ and the $\zeta$ potential was $-5.8 \mathrm{mV}$. (B) Western blotting demonstrated the expression of specific exosome marker proteins (CD9, CD63 and HSP70) in exosomes derived from samples of patients with PC and healthy controls. (C) Transmission electron microscopy images of exosomes derived from humans. Black arrows point out the typical exosomes that have a cup shape and a double-layer membrane structure. (D) The particle size of exosomes in the plasma samples of mice was 139.9 nm and the $\zeta$ potential was $-1.49 \mathrm{mV}$. (E) Western blotting demonstrated the expression of specific exosome marker proteins (CD9 and HSP70) in exosomes derived from plasma samples of mice with PC and healthy controls. (F) Transmission electron microscopy images of exosomes derived from mice. Black arrows point out the typical exosomes that have a cup shape and a double-layer membrane structure. (G) Total internal reflection fluorescence microscopy images of exmiR-21, exmiR-10b and exmiR-212-3p expression in human serum exosomes. (H) A set of TIRF microscopy images of exmiR-21 fluorescent signals from exosomes isolated from mice serum samples. PC, pancreatic cancer; HSP70, heat shock protein 70; exmiR, exosomal microRNA; TIRF, total internal reflection fluorescence. 

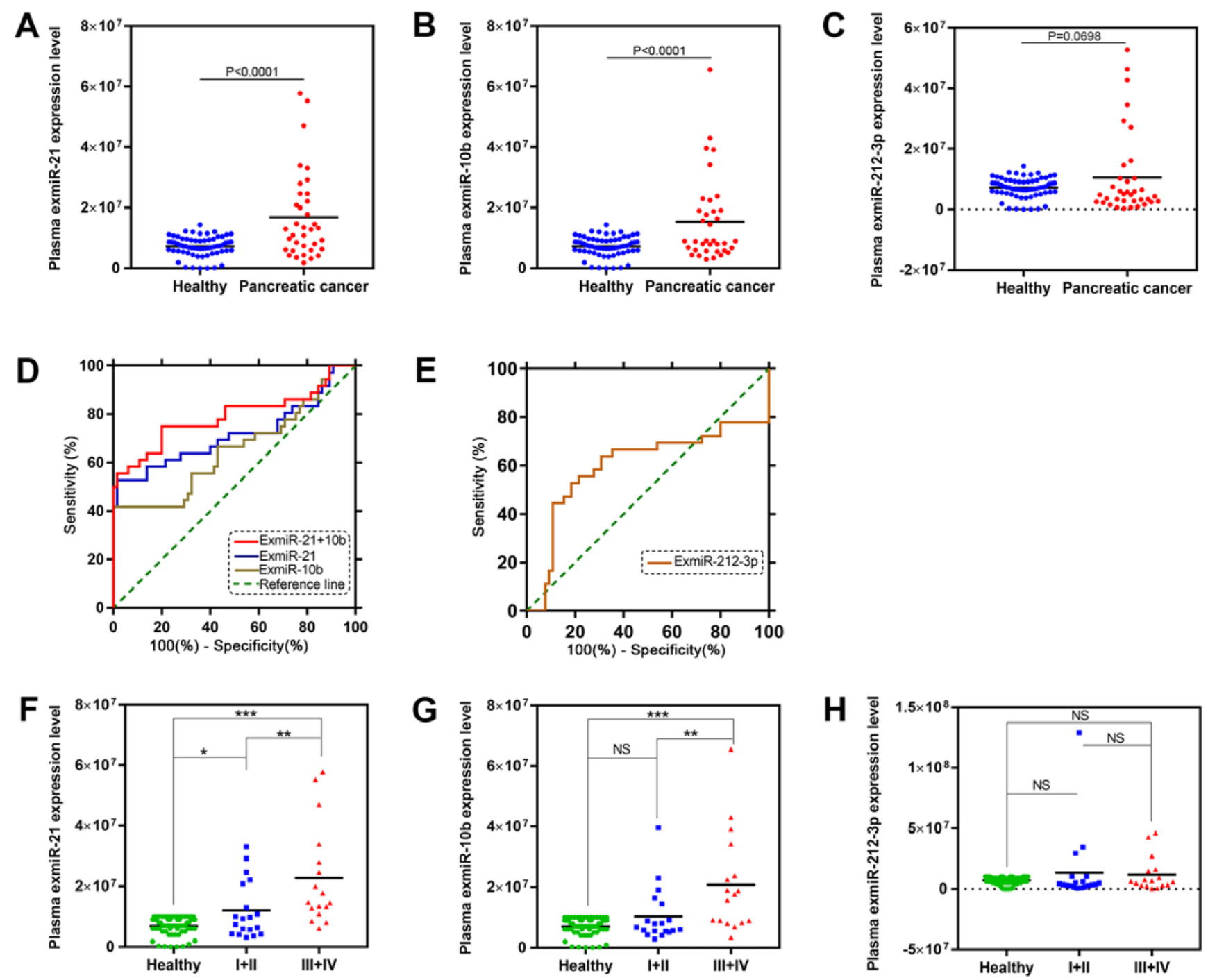

Figure 2. Comparing the expression pattern and diagnostic values of selected plasma exmiRs between patients with PC and control. The relative expression levels of (A) the exmiR-21, (B) exmiR-10b and (C) exmiR-212-3p. ROC analyses were performed among the candidate exmiRs: (D) Relative plasma exmiR-21, exmiR-10b and exmiR-21 combined with exmiR-10b levels in patients with PC $(n=36)$ and healthy controls $(n=65)$. (E) ROC curve of relative plasma exmiR-212-3p levels in patients with PC $(n=36)$ and healthy controls $(n=65)$. The patients with PC were further divided into two groups, early and advanced. Patients at stages I and II (according to the 8th American Joint Committee on Cancer) were divided into the early group, and the other patients at stages III and IV were in the advanced group. The relative expression level of $(\mathrm{F})$ exmiR-21, $(\mathrm{G})$ exmiR-10b and $(\mathrm{H})$ exmiR-212-3p was compared between the different groups of individuals. ${ }^{*} \mathrm{P}<0.05 .{ }^{* *} \mathrm{P}<0.001 .{ }^{* * *} \mathrm{P}<0.0001$. NS, no significant difference. PC, pancreatic cancer; exmiR; exosomal microRNA; ROC, receiver operating characteristic.

upregulated in patients with $\mathrm{PC}$, and the results of the present study are consistent with these findings. The diagnostic performance of these exmiRs were also compared, which revealed that exmiR-21 showed the best diagnostic performance among these three exmiRs. Furthermore, the diagnostic performance of exmiR-21 was increased when combined with exmiR-10b. $\mathrm{Wu}$ et al (41) reported that miR-212-3p plays an important role in the development of gastric cancer. MiR-212-3p is one of the top differentially expressed miRs in PC (42), and a previous study confirmed that miR-212-3p is expressed at higher levels in PC tissues compared with controls (42). Furthermore, patients with PC and higher miR-212-3p expression had a decreased overall survival compared with those with low miR-212-3p expression. Therefore, miR-212-3p was selected as a candidate $\mathrm{miR}$ for analysis in the present study. However, no significant difference was observed in the expression of miR-212-3p between patients with PC and healthy controls, and exmiR-212-3p exhibited the poorest diagnostic performance among the three exmiRs.

The results indicated that exmiR-21 could distinguish early-stage patients from both healthy individuals and patients with advanced-stage PC, indicating that exmiR-21 showed potential diagnostic value for early-stage PC.

Although the etiology of PC remains largely unclear, cigarette smoking, obesity, history of drinking alcohol and diabetes mellitus have been associated with an increased risk of PC (43). These risk factors were thus examined for any effect on the expression of the exmiRs in patients with PC in the present study. The expression levels of exmiRs were evaluated in subgroups, according to age, gender, body mass index, blood type (44), history of smoking, history of drinking and diabetes mellitus in the 36 patients with PC. No differences were observed in the levels of exmiRs in any of the subgroups. Further studies with a larger number of cases are 

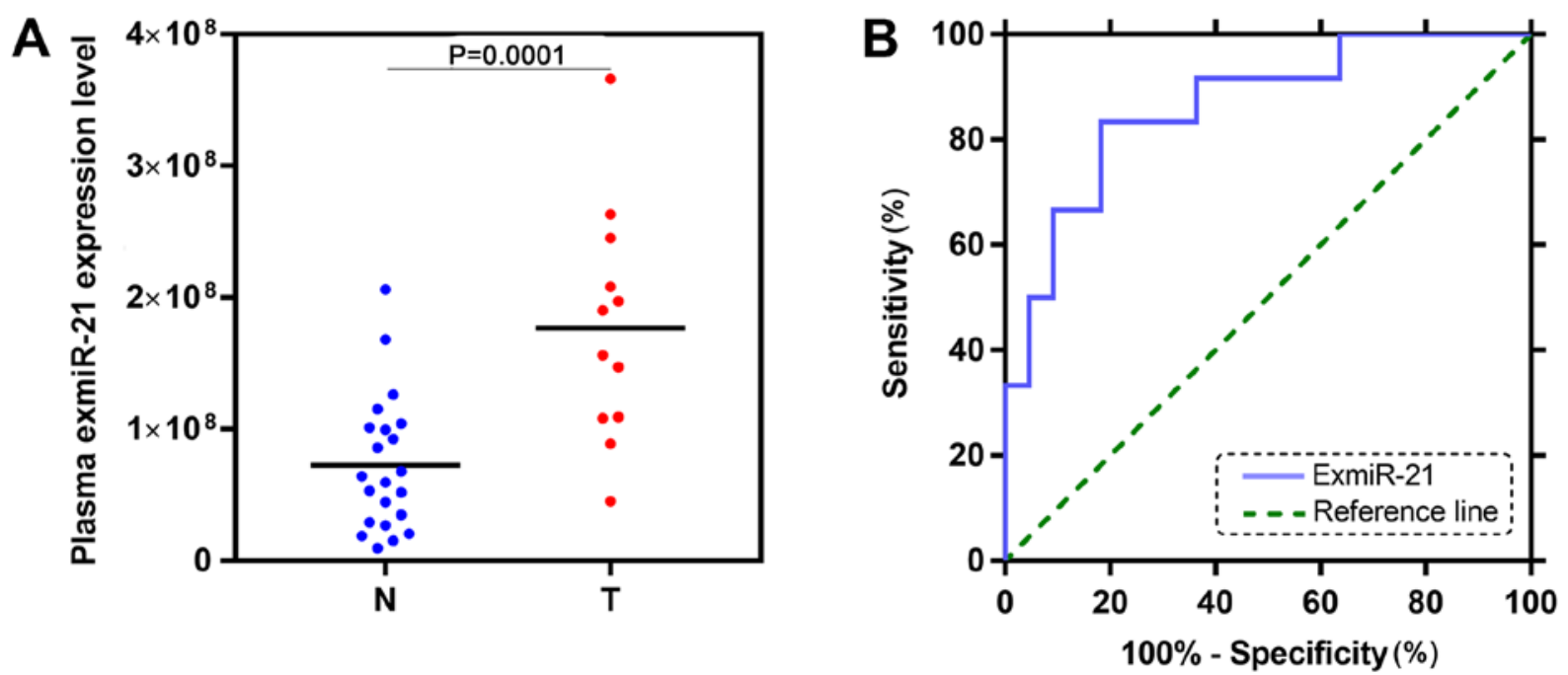

Figure 3. Expression level and diagnostic value of plasma exmiR-21 in mice. (A) Relative expression level of exmiR-21 in the plasma of mice with PC (n=12) and healthy mice $(n=22)$. (B) ROC curve of relative plasma exmiR-21 in mice with PC and the healthy controls. $\mathrm{P}=0.0005$; AUC, 0.8636 . PC, pancreatic cancer; $\mathrm{N}$, normal mice; T, mice with PC; exmiR, exosomal microRNA; ROC, receiver operating characteristics.
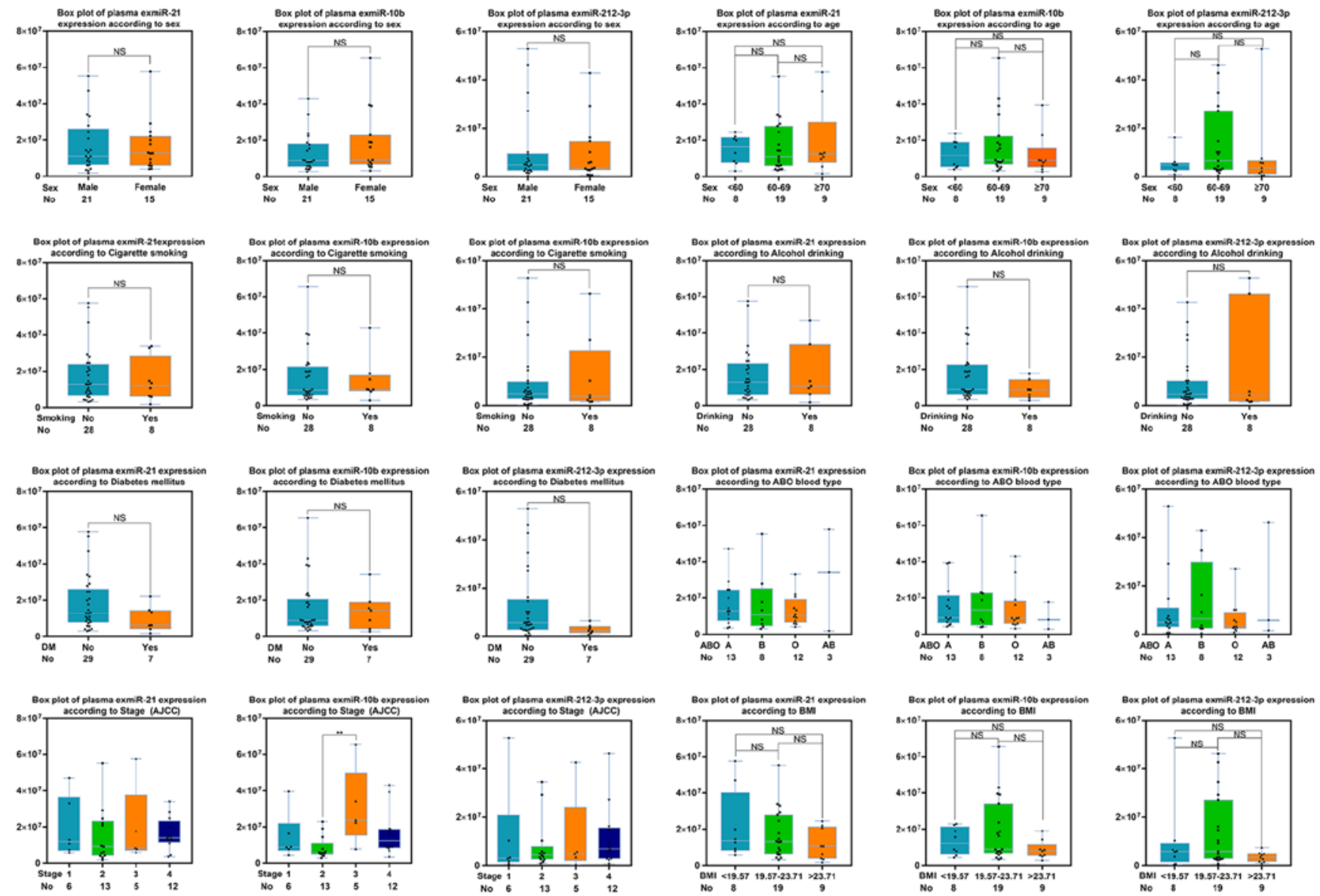

Figure 4. Association between the expression of exmiRs and clinicopathological parameters of patients with pancreatic cancer. ${ }^{* *} \mathrm{P}<0.01$. NS, no significant difference. ExmiR; exosomal microRNA; AJCC, American Joint Committee on Cancer; BMI, body mass index.

vital to evaluate the expression levels of these exmiRs based on clinical parameters.

To the best of our knowledge, the present study is the first to use the TCLN biochip to detect the expression levels of these candidate exmiRs in patients with PC, healthy indi- viduals and mice, with the aim of investigating the diagnostic value of plasma exmiRs in PC. Compared with RTq-PCR, which involves cumbersome and time-consuming methods for exosome miR extraction and detection, the TCLN biochip is a novel and efficient method of detecting RNA targets for 
the early diagnosis of cancer (6). Using the TCLN biochip, the levels of exmiRs can be easily determined in various cancer types. In the present study, the utility of using the TCLN biochip to evaluate exmiR-21 levels was demonstrated to distinguish patients with early-stage PC from healthy individuals. Although further large-scale studies are required to validate the present findings, these results suggest that this method will increase the number of patients with PC diagnosed at the early-stages, giving the opportunity to be treated with surgery and prevent disease progression.

The present study has several limitations. Due to the limited number of patients with PC in the study, a training and validation cohort could not be set. However, the plasma levels of exmiR-21 in 34 mice were validated. The present study also only compared the expression levels of the three exmiRs in patients with PC and healthy individuals; benign pancreatic disease could be a valuable inclusion (such as chronic pancreatitis, pancreatic neuroendocrine neoplasms or cystic tumors). Bloomston et al (45) suggested that miR-21 was uniquely upregulated in patients with PC compared with normal individuals and patients with chronic pancreatitis. Abue et al (46) reported that the miR-483-3p and miR-21 expression levels were significantly higher in PDAC than in normal individuals and patients with intraductal papillary mucinous neoplasm. Additionally, the present study only compared the preoperative patients with healthy individuals. Future studies should examine the levels of these exmiRs before and after treatment, such as surgery, radiotherapy, chemotherapy and molecular targeted therapy. As aforementioned, further studies with larger samples of both patients with PC and healthy individuals will be important to verify the reliability of the diagnostic performance of exmiR-21. Furthermore, other exmiRs that may have higher sensitivity and specificity in PC could also be considered. Therefore, RNA sequencing-based screening to identify PC-specific exmiRs will be conducted, in order to focus on exmiRs with the highest levels of upregulation, for further analysis.

The results of the present study demonstrated that plasma levels of exmiR-21 and exmiR-10b were increased in patients with PC compared with controls. ROC analyses demonstrated that exmiR-21 had the best diagnostic performance among the three exmiRs. ExmiR-21 was also found to discriminate patients with early-stage PC from both healthy controls and patients with advanced PC. These findings suggest that evaluating exmiR-21 using the TCLN biochip may be a useful non-invasive strategy for diagnosing early stage PC.

\section{Acknowledgements}

The authors would like to acknowledge Hangzhou Dixiang Co. Ltd. (Hangzhou, China) for their support with the tethered cationic lipoplex nanoparticle biochip technology.

\section{Funding}

The present study was supported by the Major Project of Medical Science and Technology of Zhejiang Province (grant no. WKI-ZJ-1824); the Foundation Project for Medical Science and Technology of Zhejiang province (grant no. 2018KY102); the Major Research Project of Science Technology Department of Zhejiang Province (grant no. 2019C03048).

\section{Availability of data and materials}

The datasets used and/or analyzed during the present study are available from the corresponding author upon reasonable request.

\section{Authors' contributions}

LC and SJ designed the study, performed the experiments, wrote the manuscript and prepared the figures. XP and GD were involved in the study design, and performed the experiments and data analysis. SZ collected the samples and characteristics of healthy individuals and patients. MW assisted with performing the experiment and verifying the results. All authors reviewed the manuscript, and approved the final version of the published manuscript.

\section{Ethics approval and consent to participate}

The research protocol was reviewed and approved by the Research Ethics Committee of Sir Run Run Shaw Hospital, School of Medicine, Zhejiang University (approval; no. 20170222-16). All participants or their guardians provided written informed consent for scientific research statement. All animal experiments were carried out in the animal unit of Zhejiang University according to procedures authorized and specifically approved by the institutional Ethical Committee.

\section{Patient consent for publication}

Not applicable.

\section{Competing interests}

The authors declare that they have no competing interests.

\section{References}

1. Siegel RL, Miller KD and Jemal A: Cancer statistics, 2018. CA Cancer J Clin 68: 7-30, 2018.

2. Rahib L, Smith BD, Aizenberg R, Rosenzweig AB, Fleshman JM and Matrisian LM: Projecting cancer incidence and deaths to 2030: The unexpected burden of thyroid, liver, and pancreas cancers in the United States. Cancer Res 74: 2913-2921, 2014.

3. Zhu H, Li T, Du Y and Li M: Pancreatic cancer: Challenges and opportunities. BMC Med 16: 214, 2018.

4. Goto T, Fujiya M, Konishi H, Sasajima J, Fujibayashi S, Hayashi A, Utsumi T, Sato H, Iwama T, Ijiri M, et al: An elevated expression of serum exosomal microRNA-191, - 21, -451a of pancreatic1 neoplasm is considered to be efficient diagnostic marker. BMC Cancer 18: 116, 2018.

5. Lin Y, Lin Z, Fang Z, Li H, Zhi X and Zhang Z: Plasma MicroRNA-34a as a potential biomarker for early diagnosis of esophageal cancer. Clin Lab 65, 2019.

6. Fadaka AO, Pretorius A and Klein A: Functional prediction of candidate MicroRNAs for CRC management using in silico approach. Int J Mol Sci 20: E5190, 2019.

7. Matsuzaki J and Ochiya T: Circulating microRNAs and extracellular vesicles as potential cancer biomarkers: A systematic review. Int J Clin Oncol 22: 413-420, 2017.

8. Kawaguchi T, Komatsu S, Ichikawa D, Tsujiura M, Takeshita H, Hirajima S, Miyamae M, Okajima W, Ohashi T, Imamura T, et al: circulating MicroRNAs: A next-generation clinical biomarker for digestive system cancers. Int J Mol Sci 17: E1459, 2016.

9. Salehi M and Sharifi M: Exosomal miRNAs as novel cancer biomarkers: Challenges and opportunities. J Cell Physiol 233: 6370-6380, 2018. 
10. Wang L, Zhao F, Xiao Z and Yao L: Exosomal microRNA-205 is involved in proliferation, migration, invasion, and apoptosis of ovarian cancer cells via regulating VEGFA. Cancer Cell Int 19: $281,2019$.

11. Fang JH, Zhang ZJ, Shang LR, Luo YW, Lin YF, Yuan Y and Zhuang SM: Hepatoma cell-secreted exosomal microRNA-103 increases vascular permeability and promotes metastasis by targeting junction proteins. Hepatology 68: 1459-1475, 2018.

12. Li XJ, Ren ZJ, Tang JH and Yu Q: Exosomal MicroRNA MiR-1246 promotes cell proliferation, invasion and drug resistance by targeting CCNG2 in breast cancer. Cell Physiol Biochem 44: 1741-1748, 2017.

13. Wu Y, Kwak KJ, Agarwal K, Marras A, Wang C, Mao Y, Huang X, Ma J, Yu B, Lee R, et al: Detection of extracellular RNAs in cancer and viral infection via tethered cationic lipoplex nanoparticles containing molecular beacons. Anal Chem 85: 11265-11274, 2013.

14. Lee LJ, Yang Z, Rahman M, Ma J, Kwak KJ, McElroy J, Shilo K, Goparaju C, Yu L, Rom W, et al: Extracellular mRNA detected by tethered lipoplex nanoparticle biochip for lung adenocarcinoma detection. Am J Respir Crit Care Med 193: 1431-1433, 2016.

15. Hu J, Kwak KJ, Shi J, Yu B, Sheng Y and Lee LJ: Overhang molecular beacons encapsulated in tethered cationic lipoplex nanoparticles for detection of single-point mutation in extracellular vesicle-associated RNAs. Biomaterials 183: 20-29, 2018

16. Amin MB, Greene FL, Edge SB, Compton CC, Gershenwald JE, Brookland RK, Meyer L, Gress DM, Byrd DR and Winchester DP: The eighth edition AJCC cancer staging manual: Continuing to build a bridge from a population-based to a more 'personalized' approach to cancer staging. CA Cancer J Clin 67: 93-99, 2017.

17. Si ML, Zhu S, Wu H, Lu Z, Wu F and Mo YY: MiR-21-mediated tumor growth. Oncogene 26: 2799-2803, 2007.

18. Hong L, Han Y, Zhang Y, Zhang H, Zhao Q, Wu K and Fan D: MicroRNA-21: A therapeutic target for reversing drug resistance in cancer. Expert Opin Ther Targets 17: 1073-1080, 2013.

19. Sheth S, Jajoo S, Kaur T, Mukherjea D, Sheehan K, Rybak LP and Ramkumar V: Resveratrol reduces prostate cancer growth and metastasis by inhibiting the Akt/MicroRNA-21pathway. PLoS One 7: e51655, 2012.

20. Xiong B, Cheng Y,MaL and Zhang C: MiR-21 regulates biological behavior through the PTEN/PI-3 K/Akt signaling pathway in human colorectal cancer cells. Int J Oncol 42: 219-228, 2013.

21. Qian X, Ren Y, Shi Z, Long L, Pu P, Sheng J, Yuan X and Kang C: Sequence-dependent synergistic inhibition of human glioma cell lines by combined temozolomide and miR-21 inhibitor gene therapy. Mol Pharm 9: 2636-2645, 2012.

22. Lai $X$ and Friedman A: Exosomal miRs in lung cancer: A mathematical model. PLoS One 11: e0167706, 2016.

23. Bica-Pop C, Cojocneanu-Petric R, Magdo L, Raduly L, Gulei D and Berindan-Neagoe I: Overview upon miR-21 in lung cancer: Focus on NSCLC. Cell Mol Life Sci 75: 3539-3551, 2018.

24. Wang M, Ji S, Shao G, Zhang J, Zhao K, Wang Z and Wu A: Effect of exosome biomarkers for diagnosis and prognosis of breast cancer patients. Clin Transl Oncol 20: 906-911, 2018.

25. Kim J, Siverly AN, Chen D, Wang M, Yuan Y, Wang Y, Lee H, Zhang J, Muller WJ, Liang H, et al: Ablation of miR-10b suppresses oncogene-induced mammary tumorigenesis and metastasis and reactivates tumor-suppressive pathways. Cancer Res 76: 6424-6435, 2016

26. Jin X, Chen Y, Chen H, Fei S, Chen D, Cai X, Liu L, Lin B, Su H, Zhao L, et al: Evaluation of tumor-derived exosomal miRNA as potential diagnostic biomarkers for early-stage non-small cell lung cancer using next-generation sequencing. Clin Cancer Res 23: 5311-5319, 2017.

27. Ayaz L, Görür A, Yaroğlu HY, Ozcan C and Tamer L: Differential expression of microRNAs in plasma of patients with laryngeal squamous cell carcinoma: Potential early-detection markers for laryngeal squamous cell carcinoma. J Cancer Res Clin Oncol 139: 1499-1506, 2013.

28. Stetefeld J, McKenna SA and Patel TR: Dynamic light scattering: A practical guide and applications in biomedical sciences Biophys Rev 8: 409-427, 2016.

29. Fischer K and Schmidt M: Pitfalls and novel applications of particle sizing by dynamic light scattering. Biomaterials 98: 79-91, 2016.
30. Parikh R, Mathai A, Parikh S, Chandra Sekhar G and Thomas R: Understanding and using sensitivity, specificity and predictive values. Indian J Ophthalmol 56: 45-50, 2008.

31. Simpson RJ, Lim JW, Moritz RL and Mathivanan S: Exosomes: Proteomic insights and diagnostic potential. Expert Rev Proteomics 6: 267-283, 2009.

32. Root A, Allen P, Tempst P and Yu K: Protein biomarkers for early detection of pancreatic ductal adenocarcinoma: Progress and challenges. Cancers (Basel) 10: E67, 2018.

33. Lennon AM, Wolfgang CL, Canto MI, Klein AP, Herman JM, Goggins M, Fishman EK, Kamel I, Weiss MJ, Diaz LA, et al: The early detection of pancreatic cancer: What will it take to diagnose and treat curable pancreatic neoplasia? Cancer Res 74: 3381-3389, 2018

34. Duell EJ, Lujan-Barroso L, Sala N, Deitz McElyea S, Overvad K, Tjonneland A, Olsen A, Weiderpass E, Busund LT, Moi L, et al: Plasma microRNAs as biomarkers of pancreatic cancer risk in a prospective cohort study. Int J Cancer 141: 905-915, 2017.

35. Ouyang H, Gore J, Deitz S and Korc M: MicroRNA-10b enhances pancreatic cancer cell invasion by suppressing TIP30 expression and promoting EGF and TGF- $\beta$ actions. Oncogene 36: 4952 , 2017.

36. Jamali L, Tofigh R, Tutunchi S, Panahi G, Borhani F, Akhavan S, Nourmohammadi P, Ghaderian SMH, Rasouli M and Mirzaei $\mathrm{H}$ Circulating microRNAs as diagnostic and therapeutic biomarkers in gastric and esophageal cancers. J Cell Physiol 233: 8538-8550, 2018.

37. Shan X, Zhang H, Zhang L, Zhou X, Wang T, Zhang J, Shu Y, Zhu W, Wen W and Liu P: Identification of four plasma microRNAs as potential biomarkers in the diagnosis of male lung squamous cell carcinoma patients in China. Cancer Med 7: 2370-2381, 2018.

38. Matamala N, Vargas MT, González-Cámpora R, Miñambres R, Arias JI, Menéndez P, Andrés-León E, Gómez-López G, Yanowsky K, Calvete-Candenas J, et al: Tumor microRNA expression profiling identifies circulating microRNAs for early breast cancer detection. Clin Chem 61: 1098-1106, 2015.

39. Lai X, Wang M, McElyea SD, Sherman S, House M and Korc M: A microRNA signature in circulating exosomes is superior to exosomal glypican-1 levels for diagnosing pancreatic cancer. Cancer Lett 393: 86-93, 2017.

40. Joshi GK, Deitz-McElyea S, Liyanage T, Lawrence K, Mali S, Sardar R and Korc M: Label-free nanoplasmonic-based short noncoding RNA sensing at attomolar concentrations allows for quantitative and highly specific assay of MicroRNA-10b in biological fluids and circulating exosomes. ACS Nano 9: 11075-11089, 2015.

41. Wu R, Li F, Zhu J, Tang R, Qi Q, Zhou X, Li R, Wang W, Hua D and Chen W: A functional variant at miR-132-3p, miR-212-3p, and miR-361-5p binding site in CD80 gene alters susceptibility to gastric cancer in a Chinese Han population. Med Oncol 31: 60, 2014.

42. Wu Z, Zhou L, Ding G and Cao L: Overexpressions of miR-212 are associated with poor prognosis of patients with pancreatic ductal adenocarcinoma. Cancer Biomark 18: 35-39, 2017.

43. Maisonneuve $\mathrm{P}$ and Lowenfels AB: Risk factors for pancreatic cancer: A summary review of meta-analytical studies. Int J Epidemiol 44: 186-198, 2015.

44. Li X, Xu H and Gao P: ABO blood group and diabetes mellitus influence the risk for pancreatic cancer in a population from China. Med Sci Monit 24: 9392-9398, 2018.

45. Bloomston M, Frankel WL, Petrocca F, Volinia S, Alder H, Hagan JP, Liu CG, Bhatt D, Taccioli C and Croce CM: MicroRNA expression patterns to differentiate pancreatic adenocarcinoma from normal pancreas and chronic pancreatitis. JAMA 297: 1901-1908, 2007

46. Abue M, Yokoyama M, Shibuya R, Tamai K, Yamaguchi K, Sato I, Tanaka N, Hamada S, Shimosegawa T, Sugamura K and Satoh K: Circulating miR-483-3p and miR-21 is highly expressed in plasma of pancreatic cancer. Int J Oncol 46: 539-547, 2015.

This work is licensed under a Creative Commons Attribution-NonCommercial-NoDerivatives 4.0 International (CC BY-NC-ND 4.0) License. 